\title{
ELECTRON TRANSPORT AND POINT CONTACT SPECTROSCOPY OF CUBIC DyCu
}

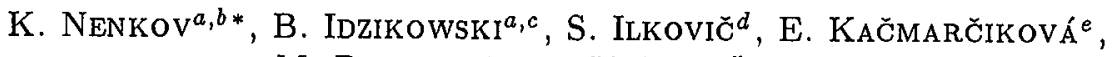 \\ M. REIFFERS ${ }^{e}$ AND K.-H. MÜLLER ${ }^{a}$ \\ ${ }^{a}$ Institut für Festkörper- und Werkstofforschung Dresden \\ P.O. Box 270016, 01171 Dresden, Germany \\ ${ }^{b}$ International Laboratory of High Magnetic Fields \\ Gajowicka 95, 53-421 Wrocław, Poland \\ cInstitute of Molecular Physics, Polish Academy of Sciences \\ Smoluchowskiego 17, 60-179 Poznań, Poland \\ ${ }^{d}$ Faculty of Sciences, University of Prešov \\ 17. novembra 1, 08078 Prešov, Slovakia \\ ${ }^{e}$ Institute of Experimental Physics, Slovak Academy of Sciences \\ Watsonova 47, 04353 Košice, Slovakia
}

\begin{abstract}
The electron transport properties and point contact spectra of melt-spun $\mathrm{DyCu}_{5}$ with the cubic phase (structure type $A u B e_{5}$, space group $F \overline{4} 3 \mathrm{~m}$ ) were investigated. It was found by ac-susceptibility measurements that below a transition temperature $T_{\mathrm{m}}=6.5 \mathrm{~K}$ this phase shows metamagnetism. Between $T_{\mathrm{m}}$ and $T_{\mathrm{C}}=14.5 \mathrm{~K}$ a spontaneous magnetization is found. Our measurements of electrical resistivity confirm the existence of two magnetic phase transitions in the compound. Furthermore, we present point contact spectra of $\mathrm{DyCu}_{5}-\mathrm{Cu}$ heterocontacts which are directly proportional to the electron-quasiparticle interaction function. Apart from a peak at about $18 \mathrm{meV}$, which is characteristic of electron-phonon interaction in pure $\mathrm{Cu}$, we observed a series of additional maxima at lower energy, which are connected with the allowed $4 f$-transitions from the ground state to excited crystalline electric field levels.
\end{abstract}

PACS numbers: 71.20.Eh, 72.15.Eb, 73.23.Ad, 73.40.Jn

\section{Introduction}

The copper rich compounds of $\mathrm{RCu}_{5}$, where $\mathrm{R}$ is a heavy rare-earth element, possess either cubic or hexagonal lattice structures [1]. The occurrence of both crystallographic structures for $\mathrm{R}$ with intermediate ionic radius is the reason that the preparation of the cubic compound is difficult. One successful preparation method is melt spinning [2]. The magnetic behaviour of cubic $\mathrm{RCu}_{5}$ compounds

\footnotetext{
*On leave from Institute of Solid State Physics, BAS, Sofia, Bulgaria.
} 
is attributed to RKKY-type indirect exchange interactions. So far, experimental work on these compounds was concerned mainly with their magnetoresistance, e.g. $\mathrm{DyCu}_{5}$ exhibits a negative magnetoresistance ratio $M R(H)=[R(H)-R(0)] / R(0)$ of about $-27 \%$ at $10 \mathrm{~K}$ in a magnetic field of $5 \mathrm{~T}$ [3], and other uncommon electron transport phenomena and crystalline electric field (CEF) effects [4, 5]. Point contact (PC) spectroscopy has proved to be a very useful experimental method for the direct study of the energy dependence of the interaction between conduction electrons and quasiparticle excitations in metals $[6,7]$. Small constrictions between two metals show deviations from Ohm's law. When both the inelastic $\left(l_{i}\right)$ and elastic $\left(l_{e}\right)$ mean free paths of the conduction electrons are larger than the contact diameter $d$, the transport of electrons through the constriction is ballistic. With an applied voltage across the contact, the electrons are accelerated when passing through the contact area. Thus, the applied voltage $V$ directly defines the energy scale for the scattering processes. The relaxation of accelerated electrons results in voltage-dependent corrections to the current. These deviations from Ohm's law are most clearly visible by a measurement of the second derivative $\mathrm{d}^{2} V / \mathrm{d} I^{2}$ of the $I-V$ characteristic. $\mathrm{d}^{2} V / \mathrm{d} I^{2}$ vs. $V$ curve is proportional to the spectral function for the interaction processes. Because of the evidence for two magnetic phase transitions in cubic $\mathrm{DyCu}_{5}$ [2] it is interesting to study the electron-quasiparticle interaction and the CEF contribution related to $4 f$-excitations for this compound by point contact spectroscopy.

\section{Experimental}

High-purity elements (Dy 99.95, Cu 99.95\%) were used to make starting ingots. Melt-spun alloys of $\mathrm{DyCu}_{5}$ were prepared by single roller technique on a copper wheel as described in Ref. [2]. The structure of the ribbons was examined by X-ray diffraction (XRD) using Co $K_{\alpha}$ radiation. ac-susceptibility was measured in a commercial Lake Shore susceptometer. Electrical resistance $R$ was determined using standard four-probe contacts in the temperature range from $5 \mathrm{~K}$ to $200 \mathrm{~K}$ and in magnetic field up to $5 \mathrm{~T}$ in conventional superconducting magnets. The heterocontacts were made by pressing a Cu needle onto the surface of $\mathrm{DyCu}_{5}$. The derivatives $\mathrm{d} V / \mathrm{d} I$ and $\mathrm{d}^{2} V / \mathrm{d} I^{2}$ of the $I-V$ characteristics were measured using a phase-sensitive technique [7] in the temperature range from $1.6 \mathrm{~K}$ up to $17 \mathrm{~K}$.

\section{Results}

The temperature dependence of the electrical resistivity of cubic $\mathrm{DyCu}_{5}$ measured with and without magnetic field is shown in Fig. 1a. The resistivity in zero field displays two anomalies, a peak and a shoulder, at low temperatures. The resistivity in an applied magnetic field of $5 \mathrm{~T}$ does not show these anomalies and it is drastically reduced below a temperature of about $75 \mathrm{~K}$. In Fig. $1 \mathrm{~b}$ the resistivity in zero field at low temperatures is compared to the magnetic susceptibility. The comparison shows that the anomalies of the resistivity are related to those of the susceptibility. Estimating characteristic temperatures from the discontinuous change of the slope of the resistivity versus temperature curves, we find the two values $T_{\mathrm{C}} \approx 13-14 \mathrm{~K}$ and $T_{\mathrm{m}} \approx 6 \mathrm{~K}$, which are in good agreement with the 

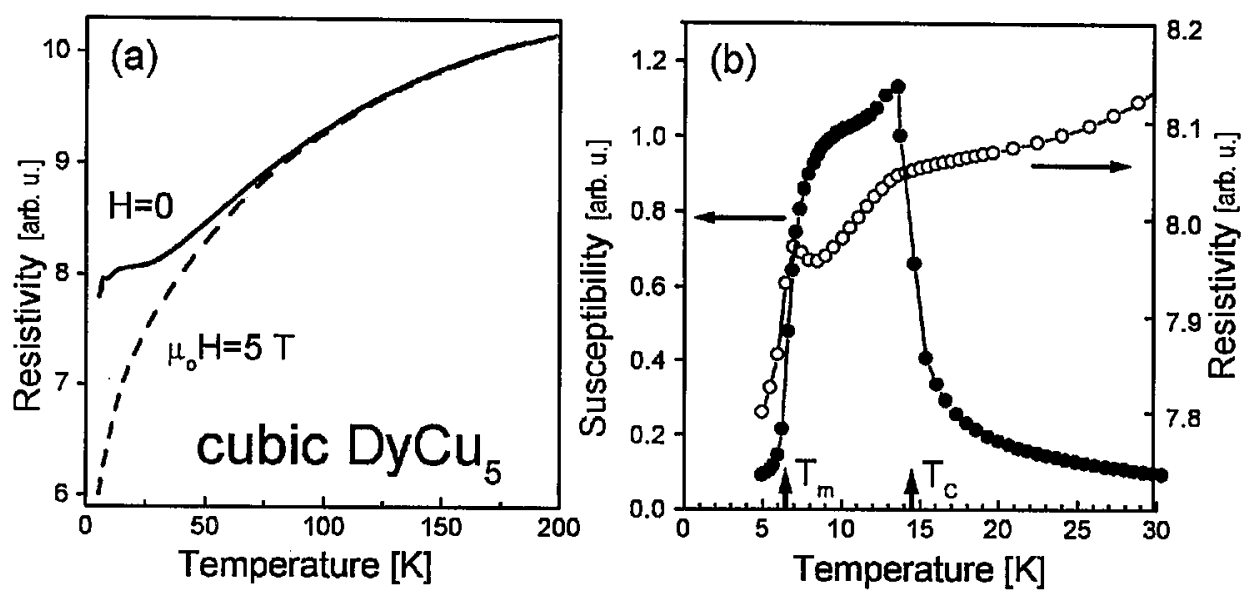

Fig. 1. Resistivity vs. temperature of cubic $\mathrm{DyCu}_{5}$ : (a) resistivity without magnetic field and in $5 \mathrm{~T}$, (b) comparison of resistivity (for $H=0$ ) with ac-susceptibility. Curie temperature $T_{\mathrm{C}}$ and metamagnetic-transition temperature $T_{\mathrm{m}}$ are indicated by arrows.

Curie temperature and the metamagnetic-transition temperature determined by magnetization measurements [2].

In Fig. 2 the PC spectra of the heterocontacts between $\mathrm{DyCu}_{5}$ and $\mathrm{Cu}$ in the ballistic regime are presented. We have observed a broad peak at about $18 \mathrm{meV}$, which is characteristic of the electron-phonon interaction function in pure $\mathrm{Cu}$ [7]. However, we do not see the characteristic maximum at $30 \mathrm{meV}$ due to phonons in pure $\mathrm{Cu}$. At low temperatures, below the metamagnetic transition (Fig. 2a), we find additional features in the spectra. There is a series of peaks at about 1, 5, 10, and a small shoulder at about $11 \mathrm{meV}$. These features are well reproducible. In order to determine the origin of the peaks, we have measured the temperature dependence of the PC spectrum for the one PC. The results are shown in Fig. 2b.
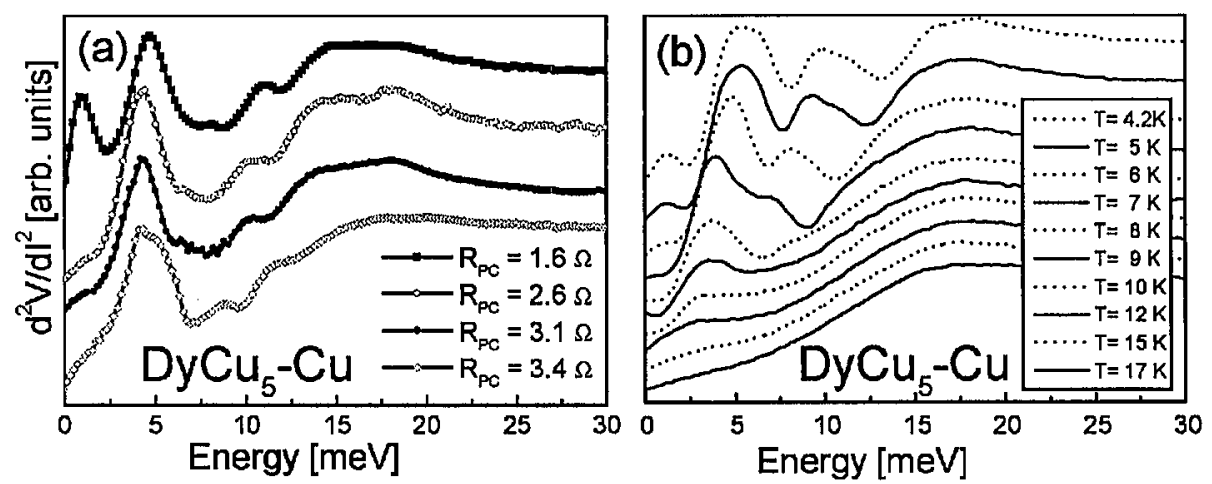

Fig. 2. Characteristic point contact spectra $\mathrm{d}^{2} V / \mathrm{d} I^{2}$ of heterocontacts DyCu${ }_{5}-\mathrm{Cu}$ in the ballistic regime: (a) for different contacts at $4.2 \mathrm{~K}$, (b) temperature dependence of the point contact spectra measured for one contact (for clarity, the curves are shifted vertically with respect to each other). 
The broad maximum at $18 \mathrm{meV}$ does not change with increasing temperature. This is an additional confirmation that it is due to inelastic electron-phonon scattering. However, the features in the low energy region of the PC spectra shift to lower energies with increasing temperature and disappear above $T_{\mathrm{C}}$. Thus, there is a clear relation between magnetic ordering and these features. We suppose that these peaks are due to the transitions of $4 f$ electrons from the CEF ground state to excited CEF levels [8]. The shift of the peaks to lower energies with increasing temperature could be explained as the manifestation of the Zeeman splitting of $\mathrm{CEF}$ doublets in magnetically ordered state.

\section{Conclusion}

Electrical resistivity measurements on melt-spun ribbons of cubic $\mathrm{DyCu}_{5}$ confirm the two phase transitions found by magnetic measurements. In point contact spectra of $\mathrm{DyCu}_{5}-\mathrm{Cu}$ heterocontacts a broad peak due to the electron-phonon interaction and a series of additional peaks at low energies is found. The temperature dependence of these peaks shows that they present the magnetic contribution to the electron-quasiparticle interaction function of $\mathrm{DyCu}_{5}$. They are related to the excitations of $4 f$ electrons to higher CEF levels.

\section{Acknowledgments}

This work was supported by the Slovak Grant Agency VEGA (grant No. 2/4176/97) and by Deutsche Forschungsgemeinschaft (SFB 463). Liquid nitrogen needed for experiments was sponsored by VSŽ Ocel s.r.o., Košice.

\section{References}

[1] K.H.J. Buschow, A.M. van Diepen, H.W. de Wijn, J. Appl. Phys. 41, 4609 (1970).

[2] K.-H. Müller, B. Idzikowski, D. Eckert, K. Nenkov, H.-J. Engelmann, A. Teresiak, M. Wolf, IEEE Trans. Magn. 33, 3565 (1997).

[3] B. Idzikowski, A. Jezierski, K. Nenkov, J. Appl. Phys. 85, 4744 (1999).

[4] K. Knorr, W. Gross, J.F. Olijhoek, K.H.J. Buschow, J. Phys. F, Metal Phys. 9, $645(1979)$.

[5] P.C.M. Gubbens, A.M. van der Kraan, K.H.J. Buschow, Physica $B$ 130, 415 (1985).

[6] I.K. Yanson, J. Low Temp. Phys. 9, 676 (1983).

[7] A.G.M. Jansen, A.P. van Gelder, P. Wyder, J. Phys. C 13, 6073 (1980).

[8] M. Reiffers, T. Saloňová, D. Gignoux, D. Schmitt, Europhys. Lett. 45, 520 (1999). 